\title{
Geração de renda, emprego e impostos no agronegócio dos estados da região sul e restante do $\mathrm{Brasil}^{\dagger}$
}

\author{
Umberto Antonio Sesso Filho* \\ Joaquim José Martins Guilhoto** \\ Rossana Lott Rodrigues $^{* *}$ \\ Antonio Carlos Moretto*** \\ Magno Rogério Gomes ${ }^{* * * *}$
}

RESUMO - O dimensionamento do agronegócio deve considerar a contribuição de cada segmento dentro da cadeia produtiva e suas relações de aquisições e vendas para outros setores. Considerando que a Matriz Insumo-Produto (MIP) constitui-se em ferramenta importante para dimensionar, com maior grau de detalhamento, as cadeias produtivas e o agregado de atividades que constituem o agronegócio, este artigo concluiu, para 2004, que o Produto Interno Bruto (PIB) do agronegócio da região sul foi de, aproximadamente, R \$ 125 bilhões, o que correspondeu a $27 \%$ do total do PIB do agronegócio brasileiro (460 bilhões de reais) e 39\% do PIB total regional ( $\mathrm{R} 322$ bilhões). O estado com maior participação no agronegócio da região foi o Rio Grande do Sul, seguido do Paraná e Santa Catarina, cada um com, respectivamente, $\mathrm{R} \$ 49, \mathrm{R} \$$ 43 e R \$ 32 bilhões. A geração de empregos do agronegócio correspondeu a um terço da força de trabalho da região sul. Em termos de geração de impostos, a região sul arrecadou $\mathrm{R} \$ 8,5$ bilhões com o agronegócio em 2004, o que correspondeu a $24 \%$ do total deste setor em todo o país.

Palavras-chave: Agronegócio. Região sul. Insumo-produto.

\section{INTRODUÇÃO}

Atualmente, o Brasil possui, aproximadamente, um quarto das terras agricultáveis do

\footnotetext{
†Os autores agradecem o apoio financeiro do CNPq e da Fundação Araucária.

* Doutor em Economia Aplicada pela Escola Superior de Agricultura Luiz de Queiroz/Universidade de São Paulo. É professor do Programa de Pós-Graduação do Departamento de Economia da Universidade Estadual de Londrina e pesquisador do CNPq. Endereço eletrônico: umasesso@uel.br.

** Doutor em Economia pela Universidade de Illinois. É professor titula do Departamento de Economia da Faculdade de de Economia, Administração e Contabilidade da Universidade de São Paulo. Endereço eletrônico: guilhoto@usp.br.

*** Doutora em Economia Aplicada pela Escola Superior de Agricultura Luiz de Queiroz/Universidade de São Paulo. É professora do Programa de Pós-Graduação do Departamento de Economia da Universidade Estadual de Londrina. Endereço eletrônico: rlott@uel.br.

**** Doutor em Economia Aplicada pela Escola Superior de Agricultura Luiz de Queiroz/Universidade de São Paulo. É professor do Programa de Pós-Graduação do Departamento de Economia da Universidade Estadual de Londrina. Endereço eletrônico: acmoretto@uel.br.

***** Graduando em Ciências Econômicas pela Universidade Estadual de Londrina e bolsista da Fundação Araucária. Endereço eletrônico: magnorg@sercomtel.com.br.
} 
mundo e a elevada tecnologia utilizada no campo faz o agronegócio brasileiro ser um setor moderno, eficiente e concorrente no cenário internacional.

O agronegócio envolve a produção, processamento e distribuição dos produtos agroalimentares com impactos diretos e indiretos sobre todos os setores da economia. Trata-se de um complexo de sistemas que tem como característica a diminuição do controle da produção pelo agricultor, uma vez que este passa a depender de terceiros para a aquisição de insumos, máquinas e equipamentos e para a comercialização e a transformação da produção (DAVIS; GOLDBERG, 1957).

O dimensionamento do agronegócio deve considerar a contribuição de cada segmento dentro da cadeia produtiva e suas relações de aquisições e vendas para outros setores. Isto faz da matriz insumo-produto uma base de dados fundamental para dimensionar, com maior grau de detalhamento, as cadeias produtivas e o agregado de atividades que participam dos sistemas que constituem o agronegócio.

A construção da matriz insumo-produto inter-regional dos estados do sul e do restante do Brasil torna possível dimensionar o agronegócio dentro de cada região. Assim, o objetivo principal deste artigo é conhecer a participação do agronegócio e de seus agregados na economia regional. Especificamente, pretende-se estimar o Produto Interno Bruto (PIB), a geração de empregos e de impostos do agronegócio dos estados da região sul e do restante do país.

Este texto está dividido em cinco seções incluindo a introdução. Na segunda seção a economia do agronegócio do Brasil e dos estados da região sul é analisada a partir de seus dados primários enquanto na terceira seção é apresentada a metodologia. A quarta seção apresenta os resultados da pesquisa e a quinta as principais conclusões do estudo.

\section{O AGRONEGÓCIO NO BRASIL}

TABELA 1 - PRODUTO INTERNO BRUTO TOTAL E DO AGRONEGÓCIO, BRASIL, 2000-2008 (EM MILHÕES DE REAIS DE 2008)

\begin{tabular}{l|c|c|c|c|c|r|r|r|r}
\hline $\begin{array}{c}\text { Agronegócio } \\
\text { e total }\end{array}$ & $\mathbf{2 0 0 0}$ & $\mathbf{2 0 0 1}$ & $\mathbf{2 0 0 2}$ & $\mathbf{2 0 0 3}$ & $\mathbf{2 0 0 4}$ & $\mathbf{2 0 0 5}$ & $\mathbf{2 0 0 6}$ & $\mathbf{2 0 0 7}$ & $\mathbf{2 0 0 8}$ \\
\hline a) Insumos & 56.788 & 59.085 & 67.737 & 76.200 & 77.258 & 69.408 & 67.545 & 76.317 & 90.025 \\
b) Agropecuária & 136.047 & 142.300 & 159.236 & 178.065 & 176.527 & 159.314 & 155.920 & 174.915 & 201.009 \\
c) Indústria & 189.258 & 187.826 & 198.756 & 204.452 & 214.781 & 215.060 & 221.115 & 230.739 & 231.261 \\
d) Distribuição & 189.812 & 192.685 & 207.418 & 215.807 & 223.189 & 215.751 & 217.939 & 232.835 & 242.199 \\
Agronegócio & 571.905 & 581.896 & 633.147 & 674.524 & 691.754 & 659.534 & 662.519 & 714.806 & 764.494 \\
Brasil & 2.500 .796 & 2.501 .656 & 2.501 .415 & 2.343 .176 & 2.446 .138 & 2.553 .054 & 2.769 .893 & 2.889 .342 & 2.889 .718 \\
Participação (\%) & $23 \%$ & $23 \%$ & $25 \%$ & $29 \%$ & $28 \%$ & $26 \%$ & $24 \%$ & $25 \%$ & $26 \%$ \\
\hline
\end{tabular}

FONTE: CEPEA (2011).

A Tabela 1 apresenta os dados do PIB total e do agronegócio do Brasil e da participa- 
ção no valor total. O agronegócio representou 26\% do PIB brasileiro em 2008, considerando que a maior parte deste foi relativa à indústria e à distribuição. A agregação de valor dos produtos agrícolas ocorre, portanto, dentro da cadeia produtiva com o processamento, transporte e adequação do produto às exigências dos consumidores. O desenvolvimento econômico do país deverá acelerar este processo, pois os consumidores exigirão produtos com maior conveniência, processamento e nível de serviço por parte dos varejistas, o que irá aumentar a participação dos elos finais das cadeias produtivas no valor adicionado dos produtos.

TABELA 2 - PRODUTO INTERNO BRUTO (PIB), POPULAÇÃO E RENDA PER CAPITA DAS GRANDES REGIÕES DO BRASIL, 2004 (EM REAIS DE 2004)

\begin{tabular}{lrrrr}
\multicolumn{1}{c}{ Região } & Produto Interno Bruto (R\$ milhão) & População & PIB per capita $(\mathbf{R} \$$ ) \\
\hline Norte & 93.423 & 14.373 .260 & 6.500 \\
Nordeste & 248.445 & 50.427 .274 & 4.927 \\
Sudeste & 970.245 & 77.374 .720 & 12.540 \\
Sul & 321.781 & 26.635 .629 & 12.081 \\
Centro-Oeste & 132.727 & 12.770 .141 & 10.394 \\
Brasil & 1.766 .621 & 181.581 .024 & 9.729 \\
\hline
\end{tabular}

Considerando que a matriz insumo-produto inter-regional dos estados da região sul e do restante do Brasil foi construída para o ano de referência de 2004, a Tabela 2 mostra o PIB, a população e o PIB per capita para a economia nacional e grandes regiões. Verificou-se que a região sudeste, que representava cerca de 55\% da economia nacional em 2004, liderou com 970 bilhões dos 1.766 bilhões de reais do PIB do país e R $\$ 12.540$ anuais de PIB per capita. As regiões sul e centro-oeste apresentaram renda per capita maior do que a média nacional enquanto as regiões norte e nordeste possuíam valores menores do que aquele em 2004.

\section{METODOLOGIA}

\subsection{CONSTRUÇÃO DO SISTEMA INTER-REGIONAL SUL - RESTANTE DO BRASIL}

Para estimar a matriz de insumo-produto nacional para o ano de 2004 foi utilizada a metodologia de Guilhoto e Sesso Filho (2005a). Posteriormente, foi usada a metodologia descrita em Guilhoto e Sesso Filho (2005b) para construir o sistema inter-regional com quatro regiões, Paraná, Santa Catarina, Rio Grande do Sul e restante do Brasil, e cinquenta e cinco setores para o ano de 2003. Os setores foram distribuídos entre 3 agregados do agronegócio neste estudo da seguinte forma: O agregado I, referente aos insumos, será especificado na sequência, agregado II - agropecuária: (1) Agricultura, silvicultura e exploração florestal e (2) Pecuária e pesca; agregado III - indústria: (6) Alimentos e bebidas, (7) Produtos do fumo, (8) Têxteis, (9) 
Artigos do vestuário e acessórios, (10) Artefatos de couro e calçados, (11) Produtos de madeira, (12) Celulose e produtos de papel, (13) Jornais, revistas e discos e (15) Álcool; agregado IV distribuição: setores de (42) a (55).

\subsection{DIMENSIONAMENTO DO AGRONEGÓCIO}

A metodologia para o cálculo do PIB do agronegócio baseia-se no trabalho de Furtuoso e Guilhoto (2003) e Furtuoso (1998), fundamentando-se na intensidade da interligação para trás e para frente da agropecuária propriamente dita. O PIB do agronegócio resulta da soma de quatro agregados principais: insumos, agropecuária, indústria e distribuição.

No cálculo do PIB do agregado I (Insumos para a Agricultura e Pecuária) são utilizadas as informações referentes aos valores dos insumos adquiridos pela Agricultura e Pecuária e que estão disponíveis nas tabelas de insumo-produto. As colunas com os valores dos insumos são multiplicadas pelos respectivos coeficientes de valor adicionado $\left(C V A_{i}\right)$. Para obter-se os Coeficientes do Valor Adicionado por setor $\left(C V A_{i}\right)$ divide-se o Valor Adicionado a Preços de $\operatorname{Mercado}^{1}\left(V A_{P M i}\right)$ pela Produção do Setor $\left(X_{i}\right)$, ou seja,

$$
C V A_{i}=\frac{V A_{P M i}}{X_{i}}
$$

Desta forma, o problema de dupla contagem, comumente apresentado em estimativas do PIB do agronegócio, quando se levam em consideração os valores dos insumos e não o valor adicionado efetivamente gerado na produção destes, é eliminado.

Tem-se então:

$$
P I B_{I k}=\sum_{i=1}^{n} \gamma_{i k} \cdot C V A_{i}
$$

em que: $k=1$ para setor agricultura, 2 para pecuária; $i=1,2, \ldots, n$ setores restantes; $P I B_{I k}=$ PIB do agregado I (insumos) para agricultura $(k=1)$ e pecuária $(k=2) ; ₹_{i k}=$ valor total do insumo do setor $i$ para a agricultura ou pecuária; $C V A_{i}=$ Coeficiente de Valor Adicionado do setor $i$.

Para o agregado I total tem-se: $P I B_{I}=P I B_{I 1}+P I B_{I 2}(3)$, em que $P I B_{I}=$ PIB do agregado I e as outras variáveis são como definidas anteriormente.

Para o agregado II (propriamente, o Setor Agricultura e Pecuária), consideram-se no cálculo os valores adicionados gerados pelos respectivos setores e subtraem-se dos valores

1 O valor adicionado a preços de mercado é obtido pela soma do valor adicionado a preços básicos com os impostos indiretos líquidos de subsídios sobre produtos, resultando na seguinte expressão: $V A_{P M}=V A_{P B}+I I L$, sendo $V A_{P M}=$ Valor Adicionado a Preços de Mercado, $V A_{P B}=$ Valor Adicionado a Preços Básicos e IIL $=$ Impostos Indiretos Líquidos. 
adicionados destes setores os valores que foram utilizados como insumos, eliminando-se o problema de dupla contagem presente em estimativas anteriores do PIB do agronegócio. Tem-se então que:

$$
P I B_{I I k}=V A_{P M k}-\sum_{i=1}^{n} z_{i k} \cdot C V A_{i}
$$

em que PIB ${ }_{I I k}=$ PIB do agregado II para agricultura $(k=1)$ e pecuária $(k=2)$ e as outras variáveis são como definidas anteriormente.

Para o agregado II total tem-se: $P I B_{I I}=P I B_{I I 1}+P I B_{I I 2}(5)$, em que PIB $I I=$ PIB do agregado II e as outras variáveis são como definidas anteriormente.

Para a definição da composição do agregado III, Indústrias de Base Agrícola, foram considerados vários indicadores como, por exemplo: a) os principais setores demandantes de produtos agrícolas, obtidos através da estimação da matriz de insumo-produto; b) as participações dos insumos agrícolas no consumo intermediário dos setores agroindustriais; e c) as atividades econômicas que efetuam a primeira, segunda e terceira transformações das matériasprimas agrícolas.

Os agregados II e III, portanto, expressam a renda ou o valor adicionado gerado por esses segmentos. No caso da estimação do agregado III (Indústrias de Base Agrícola), adotouse o somatório dos valores adicionados pelos setores agroindustriais subtraídos dos valores adicionados destes setores que foram utilizados como insumos do agregado II. Como mencionado anteriormente, esta subtração visa eliminar a dupla contagem presente em estimativas anteriores do PIB do agronegócio, ou seja:

$$
P I B_{I I I k}=\sum_{q \in k}\left(V A_{P M}-\gamma_{q k} \cdot C V A_{q}\right)
$$

em que $P I B_{I I I}=$ PIB do agregado III para agricultura $(k=1)$ e pecuária $(k=2)$ e as outras variáveis são como definidas anteriormente.

Para o agregado III total tem-se: $P I B_{I I I}=P I B_{I I I 1}+P I B_{I I I 2}(7)$, em que PIB ${ }_{I I I}=P I B$ do agregado III e as outras variáveis são como as definidas anteriormente.

No caso do agregado IV, referente à Distribuição Final, considerou-se, para fins de cálculo, o valor agregado dos setores relativos ao Transporte, Comércio e segmentos de Serviços. Do valor total obtido, destinou-se ao agronegócio apenas a parcela que corresponde à participação dos produtos agropecuários e agroindustriais na demanda final de produtos. A sistemática adotada no cálculo do valor da distribuição final do agronegócio industrial pode ser 
representada por:

$$
\begin{gathered}
D F G-I I L_{D F}-P I_{D F}=D F D \\
V A T_{P M}+V A C_{P M}+V A S_{P M}=M C \\
P I B_{I V k}=M C \cdot \frac{D F_{k}+\sum_{q \in k} D F_{q}}{D F D}
\end{gathered}
$$

em que: $D F G=$ Demanda Final Global; $I I L_{D F}=$ Impostos Indiretos Líquidos pagos pela Demanda Final; $P I_{D F}=$ Produtos Importados pela Demanda Final; $D F D=$ Demanda Final Doméstica; $V A T_{P M}=$ Valor Adicionado do setor Transporte a Preços de Mercado; $V A C_{P M}=$ Valor Adicionado do setor Comércio a Preços de Mercado; $V A S_{P M}=$ Valor Adicionado do setor Serviços a Preços de Mercado; $M C=$ Margem de Comercialização; $D F_{k}=$ Demanda Final da agricultura $(k=1)$ e pecuária $(k=2) ; D F_{q}=$ Demanda Final dos setores agroindustriais; $P I B_{I V k}=$ PIB do agregado IV para agricultura $(k=1)$ e pecuária $(k=2)$.

Para o agregado IV total tem-se: $P I B_{I V}=P I B_{I V 1}+P I B_{I V 2}(11)$, em que $P I B_{I V}=$ PIB do agregado IV.

O PIB total do agronegócio é dado pela soma dos seus agregados, ou seja:

$$
P I B_{\text {Agrk }}=P I B_{I k}+P I B_{I I k}+P I B_{I I I k}+P I B_{I V k}
$$

em que $P I B_{\text {Agrk }}=$ PIB do agronegócio para agricultura $(k=1)$ e pecuária $(k=2)$.

Para o agronegócio total tem-se: $P I B_{A g r}=P I B_{A g r 1}+P I B_{A g r 2}(13)$, em que PIB $B_{A g r}=$ PIB do agronegócio.

\section{RESULTADOS E DISCUSSÃO}

\subsection{PRODUTO INTERNO BRUTO DO AGRONEGÓCIO}

O Produto Interno Bruto (PIB) do agronegócio dos estados do sul, do restante do Brasil e do Brasil constam da Tabela 3. A região sul apresentou um PIB do agronegócio de, aproximadamente, 125 bilhões de reais em 2004, valor que correspondeu a $27 \%$ do total do PIB do agronegócio brasileiro, que foi de 460 bilhões de reais. O estado que apresentou maior participação no PIB do agronegócio da região sul foi o Rio Grande do Sul, seguido do Paraná 
e de Santa Catarina, cada um com valores de, respectivamente, $\mathrm{R} \$ 49$ bilhões, $\mathrm{R} \$ 43$ bilhões e R\$32 bilhões. Em percentuais, o PIB do agronegócio do Rio Grande do Sul correspondeu a 39\% do PIB do agronegócio da região sul e a 10,64\% do PIB do agronegócio do Brasil em 2004 (Tabela 3).

TABELA 3 - PRODUTO INTERNO BRUTO DO AGRONEGÓCIO DOS ESTADOS DA REGIÃO SUL, DO RESTANTE DO BRASIL E BRASIL, 2004 (EM MILHÕES DE REAIS DE 2004)

\begin{tabular}{l|r|r|rrr|r}
\hline Agregado/Região & Paraná & Santa Catarina & Rio Grande do Sul & Região Sul & Restante do Brasil & Brasil \\
\hline Insumos & 1.918 & 830 & 1.841 & 4.589 & 16.030 & 20.619 \\
Agropecuária & 13.275 & 7.271 & 13.642 & 34.188 & 86.639 & 120.827 \\
Indústria & 12.688 & 11.218 & 12.804 & 36.710 & 96.658 & 133.368 \\
Distribuição & 15.358 & 12.985 & 20.727 & 49.070 & 136.569 & 185.639 \\
Agronegócio & 43.240 & 32.304 & 49.014 & 124.557 & 335.895 & 460.452 \\
PIB Região & 108.699 & 70.208 & 142.874 & 321.781 & 1.444 .840 & 1.766 .621 \\
Relação (\%) & $40 \%$ & $46 \%$ & $34 \%$ & $39 \%$ & $23 \%$ & $26 \%$ \\
\hline
\end{tabular}

FONTE: Cálculos dos autores.

Fazendo a mesma relação para o Paraná, este foi responsável por 34\% do PIB do agronegócio da Região Sul e por 9\% do agronegócio do Brasil, enquanto Santa Catarina participou com $26 \%$ do PIB do agronegócio da região Sul e com 7\% do PIB do território nacional (Tabela 3).

É interessante ressaltar que o agregado IV - Distribuição foi o que mais contribui com o valor do PIB do agronegócio em 2004 em todas as regiões, com média de 40\%. (Tabela 3). Em seguida, se destacou o agregado III - Indústria, exceto para o Rio Grande do Sul e o Paraná onde o Agregado II - Agropecuária foi mais importante. Considerando que, de forma geral, o Brasil ainda poderia agregar valor aos produtos que exporta, estes resultados indicam que existe nestes dois estados grande possibilidade de elevar a renda gerada no agronegócio via agregação de valor aos produtos por industrialização.

\subsection{GERAÇÃO DE EMPREGOS NO AGRONEGÓCIO}

Os resultados da geração de empregos em cada segmento do agronegócio estão na Tabela 4. A região sul empregou no agronegócio, aproximadamente, oito milhões e setecentas mil pessoas, o que representou um terço do pessoal ocupado total, evidenciando a grande importância social deste para a região. Este valor correspondeu a cerca de 25\% do total de pessoas empregadas com o agronegócio em todo o Brasil. Ao distribuir esse valor entre os estados, constatou-se que o Rio Grande do Sul foi o que mais empregou pessoas no agronegócio, totalizando três milhões e setecentas mil pessoas, ou seja, $42 \%$ do total de empregados gerados pelo agronegócio na região sul.

O estado do Paraná é o segundo colocado na geração de empregos pelo agronegócio, 
ocupando dois milhões e oitocentas mil pessoas, cerca de $28 \%$ de todos os empregos do estado, o correspondente a $32 \%$ do total de pessoas empregadas pelo agronegócio na região sul. Em Santa Catarina, os empregos gerados no agronegócio somaram 2,2 milhões, perfazendo $38 \%$ dos empregos do estado e $25 \%$ dos empregos do agronegócio da região sul. Em todas as regiões o agregado II - Agropecuária foi o maior gerador de emprego do agronegócio, sendo responsável por, aproximadamente, em média, 52\% do pessoal ocupado, em 2004 (Tabela 4).

TABELA 4 - PESSOAL OCUPADO NOS AGREGADOS DO AGRONEGÓCIO DOS ESTADOS DA REGIÃO SUL, NO RESTANTE DO BRASIL E BRASIL, 2004 (EM MILHARES DE POSTOS DE TRABALHO)

\begin{tabular}{lr|r|rr|rrr}
\hline Agregado/Região & Paraná & Santa Catarina & Rio Grande do Sul & Região Sul & Restante do Brasil & Brasil \\
\hline Insumos & 75 & 32 & 62 & 169 & 534 & 703 \\
Agropecuária & 1.580 & 885 & 2.024 & 4.489 & 14.385 & 18.874 \\
Indústria & 506 & 723 & 611 & 1.840 & 4.446 & 6.286 \\
Distribuição & 672 & 565 & 1.020 & 2.258 & 6.526 & 8.784 \\
Agronegócio & 2.833 & 2.205 & 3.718 & 8.756 & 25.890 & 34.646 \\
Região & 10.135 & 5.774 & 10.726 & 26.635 & 154.946 & 181.581 \\
Relação (\%) & $28 \%$ & $38 \%$ & $35 \%$ & $33 \%$ & $17 \%$ & $19 \%$ \\
\hline
\end{tabular}

FONTE: Cálculos dos autores.

\subsection{GERAÇÃO DE IMPOSTOS INDIRETOS LÍQUIDOS}

Ao verificar os impostos oriundos do agronegócio, mostrados nas Tabelas 5 e 6, evidencia-se que a região sul foi responsável por um total de $\mathrm{R} \$ 8,5$ bilhões, perfazendo $24 \%$ do total deste setor em todo o país em 2004. O Rio Grande do Sul, estado que possui o maior PIB do agronegócio na região sul, foi o que mais arrecadou impostos em função desta atividade, $\mathrm{R} \$ 3,6$ bilhões, valor equivalente a 42\% do total de arrecadações da região sul para o agronegócio. No Brasil, a arrecadação do Rio Grande do Sul correspondeu a 10\% do total de contribuições oriundas do agronegócio brasileiro.

TABELA 5 - IMPOSTOS INDIRETOS LÍQUIDOS (IIL) GERADOS PELO AGRONEGÓCIO DOS ESTADOS DA REGIÃO SUL, DO RESTANTE DO BRASIL E BRASIL, 2004 (EM MILHÕES DE REAIS DE 2004)

\begin{tabular}{lr|rrrrrr}
\hline Agregado/Região & Paraná & Santa Catarina & Rio Grande do Sul & Região Sul & Restante do Brasil & Brasil \\
\hline Insumos & 186 & 38 & 165 & 389 & 2.100 & 2.489 \\
Agropecuária & 648 & 431 & 687 & 1.767 & 3.866 & 5.633 \\
Indústria & 1.434 & 1.085 & 1.870 & 4.389 & 12.698 & 17.088 \\
Distribuição & 585 & 461 & 890 & 1.936 & 7.512 & 9.448 \\
Total & 2.853 & 2.015 & 3.612 & 8.481 & 26.176 & 34.657 \\
\hline
\end{tabular}

FONTE: Cálculos dos autores.

O Paraná foi o segundo colocado na arrecadação de impostos gerados pelo agronegócio em 2004, chegando a um montante de $\mathrm{R} \$ 2,85$ bilhões, o que correspondeu a 33\% do total arrecadado pela região sul e $8 \%$ do total dos impostos gerados pelo agronegócio brasileiro. O 
estado de Santa Catarina foi o que menos arrecadou impostos provenientes do agronegócio, $\mathrm{R} \$ 2$ bilhões, o qual correspondeu a 24\% dos impostos arrecadados do agronegócio da região sul e a 5,8\% dos impostos gerados por todo setor no país (Tabela 5).

É importante notar que, apesar do agregado IV - Distribuição ter sido o que mais contribui para o valor do PIB do agronegócio em todos os estados da região, foi o agregado III - Indústria o que mais participou na geração de impostos em todas as regiões. Assim, na Tabela 6, pode-se observar que, para Santa Catarina, o agregado III foi responsável por 54\% dos impostos do agronegócio arrecadados no estado em 2004, enquanto para o Rio Grande do Sul e o Paraná, esta participação foi de $52 \%$ e $50 \%$, respectivamente.

No que se refere à participação dos impostos no valor adicionado dos agregados, os resultados mostraram que os agregados I e III foram os mais penalizados pelos impostos em 2004, uma vez que arcaram, proporcionalmente, com o dobro de impostos pagos pelos agregados II e IV.

TABELA 6 - PARTICIPAÇÃO DOS AGREGADOS NO VALOR DE IMPOSTOS INDIRETOS LÍQUIDOS (IIL) DO AGRONEGÓCIO DOS ESTADOS DA REGIÃO SUL, DO RESTANTE DO BRASIL E BRASIL, $2004(\mathrm{EM} \%)$

\begin{tabular}{l|rrrrrr|r}
\hline Agregado/Região & Paraná & Santa Catarina & Rio Grande do Sul & Região Sul & Restante do Brasil & \multicolumn{1}{c}{ Brasil } \\
\hline Insumos & 7 & 2 & 5 & 5 & 8 & 7 \\
Agropecuária & 23 & 21 & 19 & 21 & 15 & 16 \\
Indústria & 50 & 54 & 52 & 52 & 49 & 49 \\
Distribuição & 20 & 23 & 25 & 23 & 29 & 27 \\
Total & 100 & 100 & 100 & 100 & 100 & 100 \\
\hline
\end{tabular}

FONTE: Cálculos dos autores.

\section{CONCLUSÕES}

A região sul produziu um Produto Interno Bruto do agronegócio em 2004 de cerca de $\mathrm{R} \$ 125$ bilhões, o que correspondeu a 27\% do total do PIB do agronegócio brasileiro (460 bilhões de reais) e 39\% do PIB total regional ( $\$ 322$ bilhões). O estado com maior participação no agronegócio da região sul foi o Rio Grande do Sul, seguido pelo Paraná e Santa Catarina, cada um com, respectivamente, $\mathrm{R} \$ 49, \mathrm{R} \$ 43$ e $\mathrm{R} \$ 32$ bilhões. A geração de empregos do agronegócio correspondeu a um terço da força de trabalho da região sul, o que reforça a importância social desse macrossetor em 2004.

Em termos de geração de impostos, a região sul arrecadou $\mathrm{R} \$ 8,5$ bilhões de reais com o agronegócio em 2004, o que correspondeu a $24 \%$ do total deste setor em todo o país. O Rio Grande do Sul respondeu por 42\% deste valor, enquanto o Paraná e Santa Catariana contribuíram com $32 \%$ e $25 \%$, respectivamente.

A renda, o emprego e os impostos gerados pelo agronegócio dos três estados da re- 
gião sul são informações importantes para nortear a atuação de governos e empresas na adoção de políticas que venham a permitir maior agregação de valor e geração de empregos nas cadeias produtivas que o compõem.

O agregado I - Insumos foi o que gerou maior valor adicionado por pessoa ocupada, ao mesmo tempo em que foi um dos agregados que mais sofreu com a carga tributária, juntamente com o agregado III - Indústria, em 2004. Considerando a participação de cada um dos agregados no agronegócio, deveria se analisar a possibilidade de redução de impostos para estes elos da cadeia produtiva, a fim de estimular a produção e a queda dos preços dos produtos.

\section{REFERÊNCIAS}

CEPEA. Disponíevl em: < http://www.cepea.esalq.usp.br/pib/other/pib_agronegocio_1994_ 05.xls>. Acesso em: 29/3/2011.

DAVIS, J. H.; GOLDBERG, R. A concept of agribusiness. Boston: Harvard University, 1957.

FURTUOSO, M. O produto interno bruto do complexo agroindustrial brasileiro. Tese (Doutorado em Economia Aplicada) - Escola Superior de Agricultura Luiz de Queiroz, Universidade de São Paulo, São Paulo, 1998.

FURTUOSO, M. C. O.; GUILHOTO, J. J. M. Estimativa e mensuração do produto interno bruto do agronegócio da economia brasileira - 1994 a 2000. Revista Brasileira de Economia e Sociologia Rural, v. 43, n. 4, 2003.

IBGE. Contas regionais do Brasil. Disponível em: <http://www.ibge.gov.br>. Acesso em: $29 / 3 / 2011$.

MORETTOO, A. C., RODRIGUES, R. L., SESSO FILHO, U. A., MAIA, K. O Paraná na dinâmica da renda do sistema inter-regional sul-restante do Brasil. Economia \& Tecnologia, v. 15, p. 65-75, 2008.

SESSO FILHO, U. A., RODRIGUES, R. L., MORETTO, A. C. Produção e emprego no sistema inter-regional sul-restante do Brasil: uma aplicação da matriz de insumo-produto. Revista Paranaense de Desenvolvimento, v. 112, p. 93-110, 2007. 